\title{
ESTUDIO DEL MOJADO Y CARACTERIZACIÓN SUPERFICIAL POR MICROSCOPÍA DE BARRIDO LASER CONFOCAL DE CHAPAS DE MADERA OBTENIDAS POR DESENROLLO
}

\author{
WETTABILITY STUDY AND SURFACE CHARACTERIZACION BY CONFOCAL LASER \\ SCANNING MICROSCOPY OF ROTARY-PEELED WOOD VENEERS
}

\author{
Gonzalo Vázquez ${ }^{1}$, Cristina Galiñanes ${ }^{1}$, M. Sonia Freire ${ }^{1}$, G. Antorrena ${ }^{1}$, Julia González-Alvarez ${ }^{1}$
}

\section{RESUMEN}

El objetivo de este trabajo fue estudiar las propiedades superficiales de chapas obtenidas por desenrollo de cinco especies de maderas duras: okume, ayous, chopo, fromager y eucalipto, y comparar el comportamiento de ambas caras de las chapas. Se han determinado los ángulos de contacto de equilibrio y se ha determinado la energía libre superficial de la madera así como sus componentes polar y dispersa. Asimismo, mediante microscopía de barrido láser confocal se ha evaluado la rugosidad superficial. La energía libre superficial osciló entre $49-64 \mathrm{~mJ} / \mathrm{m}^{2}$ y disminuyó en el orden okume $>$ fromager $>$ chopo $>$ ayous $\sim$ eucalipto, lo que indica un descenso de la capacidad de mojado en ese orden. El okume con el mayor valor de la energía libre superficial y los menores valores del ángulo de contacto de equilibrio se confirmó como la especie con las mejores propiedades de mojado, siendo además la que presentó los mayores valores de los parámetros de rugosidad superficial. Comparando las propiedades superficiales para las dos caras de las chapas se encontró que dependieron de la especie, pero en general, con la excepción de la rugosidad, las diferencias no fueron muy significativas siendo los mayores cambios para las chapas de okume que presentaron un mejor mojado en su cara interior.

Palabras claves: Chapas de madera de desenrollo, mojado, ángulo de contacto, energía libre superficial, microscopía de barrido láser confocal, rugosidad

\begin{abstract}
The aim of this work was to study the surface properties of rotary-peeled veneers of five species of hardwoods: okume, ayous, poplar, fromager and eucalyptus and to compare the behavior of both sides of the veneers. The equilibrium contact angles were determined together with the wood surface free energy and its polar and disperse components. Additionally, surface roughness was evaluated using confocal laser scanning microscopy. Surface free energy ranged from $49-64 \mathrm{~mJ} / \mathrm{m}^{2}$ and decreased in the order okume $>$ fromager $>$ poplar $>$ eucalyptus $\sim$ ayous, indicating a decrease in wettability in that order. Okume wood with the highest value of the surface free energy and the lowest values of the equilibrium contact angle was confirmed as the species with the best wetting properties, and also presented the highest values of surface roughness parameters. In general, there were not significant differences between the properties of both sides of the veneers, except for roughness. The largest differences were obtained for okume veneers whose interior side showed higher wettability.
\end{abstract}

Keywords: Rotary-peeled veneers, wettability, contact angle, surface free energy, confocal laser scanning microscopy, roughness 


\section{INTRODUCCIÓN}

En la preparación de tableros de madera, el grado de enlace adhesivo-madera depende de varios factores tales como la especie de madera, sus propiedades físico-químicas, los tratamientos superficiales, las propiedades del adhesivo y las condiciones de formación de la línea de cola. En particular, las propiedades superficiales de la madera determinan la calidad de la unión adhesivo-madera y, en consecuencia, las propiedades mecánicas del producto final. Por otra parte, se ha encontrado que los tratamientos mecánicos cambian las características químicas y morfológicas de la superficies sólidas (Liptakova et al. 1995, Gindl et al. 2001, Piao et al. 2010) por lo que la influencia de los procesos de mecanizado en las propiedades superficiales de la madera ha de ser considerada. Los métodos para caracterizar las propiedades superficiales de la madera se pueden clasificar en tres grandes grupos: microscópicos, que proporcionan información sobre la morfología superficial, espectroscópicos, que informan sobre la química superficial y termodinámicos, que proporcionan información sobre la energía superficial (Tshabalala 2005).

En el presente trabajo se han estudiado comparativamente las propiedades superficiales de chapas de cinco especies de maderas duras obtenidas por desenrollo y empleadas en la fabricación de tableros contrachapados: okume, ayous, chopo, fromager y eucalipto. Las propiedades termodinámicas superficiales se han determinado por análisis de ángulos de contacto y se ha empleado el método de Owens-Wendt-Rabel-Kaelble (Owens y Wendt 1969, Kaelble 1970) para determinar la energía libre superficial. Las propiedades físicas de la superficie de la madera se han evaluado mediante microscopía de barrido láser confocal (CLSM). Adicionalmente, se ha analizado en todos los casos la influencia del proceso de desenrollo en las características superficiales de las dos caras de las chapas.

\section{MATERIALES Y MÉTODOS}

\section{Materiales}

Se han empleado chapas de madera obtenidas por desenrollo de las siguientes especies de maderas duras destinadas a la fabricación de tableros contrachapados: okume (Aucoumea klaineana) (humedad: 9,5\% en base húmeda (b.h.); espesor chapa: $0,53 \mathrm{~mm}$ ), ayous (Triplochiton scleroxylon) (humedad: 9,8\% b.h.; espesor chapa: 0,80 mm), chopo (Populus alba) (humedad: 9,1\% b.h.; espesor chapa: 1,08 mm), fromager (Ceiba pentandra) (humedad: $9,7 \%$ b.h.; espesor chapa: $0,88 \mathrm{~mm}$ ) y eucalipto (Eucalyptus globulus) (humedad: 9,2\% b.h.; espesor chapa: $0,84 \mathrm{~mm}$ ). Todas las chapas fueron suministradas por el Grupo Losan (A Coruña, España).

\section{METODOLOGÍA}

\section{Medida de ángulos de contacto}

Se seleccionaron aleatoriamente tres chapas de cada una de especies de madera: okume, ayous, chopo, fromager y eucalipto. Los líquidos de prueba empleados fueron agua, etilenglicol, formamida y diyodometano. En cada chapa, se realizaron con cada disolvente ocho medidas del ángulo de contacto por cada una de las caras: la cara orientada hacia el exterior del tronco del árbol y sobre la que no incide la cuchilla en el proceso de desenrollo, que denominamos cara exterior y la cara interior orientada hacia el interior del tronco sobre la que incide la cuchilla en el proceso de desenrollo, que denominamos interior.

La medida de los ángulos de contacto se ha realizado según el método de la gota sésil considerado el más adecuado para investigaciones sobre madera (Scheikl y Dunky 1998, de Meijer et al. 2000, Gindl et al. 2001, Gindl y Tschegg 2002). Se ha empleado un equipo de medida de ángulos de contacto Dataphysics OCA 15 Plus, que puede registrar 25 imágenes/s. En cada experimento se depositó una gota 
$(10 \mu \mathrm{L})$ del líquido sobre la superficie de la chapa y la dirección de observación fue perpendicular a la dirección de la fibra en aquélla. Los ángulos de contacto se calcularon mediante el método de YoungLaplace empleando el software de análisis de imágenes SCA 20 de Dataphysics. Se realizaron medidas del ángulo de contacto cada segundo durante los primeros $20 \mathrm{~s}$, cada $10 \mathrm{~s}$ hasta los $120 \mathrm{~s}$ y cada $30 \mathrm{~s}$ hasta los $240 \mathrm{~s}$.

\section{Determinación del ángulo de velocidad de mojado constante (cwra)}

Los ángulos de contacto se evaluaron empleando el método diferencial (Nussbaum 1999). De acuerdo con este modelo, el proceso de mojado en la madera se puede dividir en dos fases: una fase de extensión, en la que el diámetro de la gota aumenta tan rápidamente que la velocidad de cambio del ángulo de contacto o velocidad de mojado $(\mathrm{d} \theta / \mathrm{dt}$, siendo $\theta$ el ángulo de contacto formado entre el líquido y el sólido) es relativamente rápida y una etapa de penetración, en la cual la velocidad de cambio del ángulo de contacto es aproximadamente constante. Si se representa la velocidad de mojado frente al tiempo, se puede determinar el valor del ángulo de contacto correspondiente a velocidad de mojado constante, que se conoce como ángulo de velocidad de mojado constante (cwra). Para cada especie, cara de la chapa y disolvente el cwra se ha determinado como la media de 24 determinaciones.

Para cada especie y líquido de prueba se ha analizado la existencia de diferencias significativas entre los valores medios del cwra de las caras interior y exterior aplicando el t-test para un nivel de confianza del 95\% en el software Statgraphics Plus 5.1

\section{Determinación de la energía libre superficial}

La energía libre superficial se ha determinado mediante el método de Owens-Wendt-Rabel-Kaelble (Owens y Wendt 1969, Kaelble 1970), que expresa la energía libre superficial del líquido $\left(\gamma_{\mathrm{LV}}\right)$ y del sólido $\left(\gamma_{\mathrm{SV}}\right)$ como la suma de sus componentes dispersa y polar:

$$
\begin{aligned}
& \gamma_{L V}=\gamma_{L V}{ }^{d}+\gamma_{L V}{ }^{p} \\
& \gamma_{S V}=\gamma_{S V}{ }^{d}+\gamma_{S V}{ }^{p}
\end{aligned}
$$

donde $\gamma_{\mathrm{LV}}{ }^{\mathrm{d}} \mathrm{y} \gamma_{\mathrm{SV}}{ }^{\mathrm{d}}$ son las componentes dispersas de la energía libre superficial del líquido y del sólido, respectivamente, $\mathrm{y} \gamma_{\mathrm{LV}}{ }^{\mathrm{p}}$ y $\gamma_{\mathrm{SV}}{ }^{\mathrm{p}}$ son las componentes polares de la energía libre superficial del líquido y del sólido, respectivamente.

Por otra parte, la energía libre interfacial sólido-líquido $\left(\gamma_{\mathrm{SL}}\right)$ puede calcularse como la media geométrica de las contribuciones del líquido y del sólido:

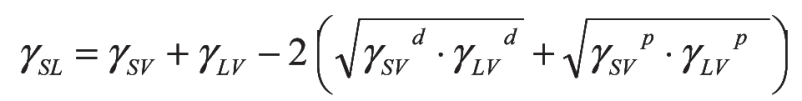

Sustituyendo $\gamma_{\mathrm{SL}}$ en la ecuación de Young (ecuación 4)

$$
\gamma_{\mathrm{LV}} \cos \theta=\gamma_{\mathrm{SV}}-\gamma_{\mathrm{SL}}
$$

se obtiene que:

$$
\gamma_{\mathrm{LV}}(1+\cos \theta)=2 \sqrt{\gamma_{\mathrm{SV}}{ }^{\mathrm{P}} \gamma_{\mathrm{LV}}{ }^{\mathrm{P}}}+2 \sqrt{\gamma_{\mathrm{SV}}{ }^{\mathrm{d}} \gamma_{\mathrm{LV}}{ }^{\mathrm{d}}}
$$


Linearizando la ecuación 5, podemos obtener las componentes polar $\left(\gamma_{\mathrm{SV}}^{\mathrm{p}}\right)$ y dispersa $\left(\gamma_{\mathrm{SV}}{ }^{\mathrm{d}}\right)$ de la energía libre superficial del sólido como la pendiente y la ordenada en el origen de la ecuación 6 , respectivamente, cuya suma da como resultado el valor de la energía libre superficial. Se han empleado cuatro líquidos de prueba cuyos valores de la tensión superficial $\left(\gamma_{\mathrm{LV}}\right)$ y de sus componentes polar $\left(\gamma_{\mathrm{LV}}^{\mathrm{p}}\right)$ y dispersa $\left(\gamma_{\mathrm{LV}}{ }^{\mathrm{d}}\right)$ son conocidos (Tabla 1): el agua en la que predomina la componente polar de la tensión superficial, el diyodometano en el que la componente polar es cero y la formamida y el etilenglicol en los que la componente dispersa representa más del $60 \%$.

$$
\frac{0,5 \gamma_{L V}(1+\cos \theta)}{\left(\gamma_{L V}^{d}\right)^{1 / 2}}=\sqrt{\gamma_{S V}{ }^{p}}\left(\frac{\gamma_{L V}^{p}}{\gamma_{L V}^{d}}\right)^{1 / 2}+\sqrt{\gamma_{S V}{ }^{d}}
$$

Tabla 1. Tensión superficial y componentes dispersa y polar de los líquidos de prueba.

\begin{tabular}{|c|c|c|c|}
\hline Disolvente & $\gamma_{\mathbf{L V}}\left(\mathbf{m J} / \mathbf{m}^{2}\right)$ & $\boldsymbol{\gamma}_{\mathbf{L V}}{ }^{\mathbf{d}}\left(\mathbf{m J} / \mathbf{m}^{\mathbf{2}}\right)$ & $\boldsymbol{\gamma}_{\mathbf{L V}}{ }^{\mathbf{p}}\left(\mathbf{m J} / \mathbf{m}^{\mathbf{2}}\right)$ \\
\hline Agua & 72,8 & 21,8 & 51,0 \\
\hline Formamida & 58,0 & 39,0 & 19,0 \\
\hline Etilenglicol & 48,0 & 29,0 & 19,0 \\
\hline Diyodometano & 50,8 & 50,8 & 0 \\
\hline
\end{tabular}

\section{Caracterización superficial de las chapas por microscopía de barrido láser confocal (CLSM)}

Muestras de las caras exterior e interior de chapas de cada una de las especies de madera estudiadas, previamente sombreadas con oro en un equipo Bio-Rad E5000 Sputted Coater, se analizaron por microscopía confocal utilizando un microscopio TCS SP2 Leica provisto de una lente HC PL APO $10 \mathrm{X} / 0.40$. En cada muestra de $2 \mathrm{~cm} \times 2 \mathrm{~cm}$ se han seleccionado aleatoriamente cinco campos de medición, de dimensiones 2,0 $\mathrm{mm}$ (longitud) x 2,0 $\mathrm{mm}$ (anchura) x 0,5 $\mathrm{mm}$ (espesor). Las secciones ópticas se obtuvieron usando intervalos de $2,5 \mu \mathrm{m}$ en la dirección z. Los resultados se analizaron utilizando el software Confocal de Leica para obtener imágenes topográficas de la superficie, reconstrucciones tridimensionales, los perfiles de rugosidad a lo largo de una trayectoria y los parámetros de rugosidad media aritmética $(\mathrm{Ra})$ y media cuadrática (RMS), calculados de acuerdo con el estándar DIN EN ISO 4287 mediante las ecuaciones 7 y 8.

$$
\begin{aligned}
\mathrm{Ra} & =\frac{1}{\mathrm{~L}} \int_{0}^{\mathrm{L}}|\mathrm{z}(\mathrm{x})| \mathrm{dx} \quad \text { con } \mathrm{z}(\mathrm{x})=\mathrm{z}_{\mathrm{i}}-\overline{\mathrm{z}} \\
\mathrm{RMS} & =\sqrt{\frac{1}{\mathrm{~L}} \int_{0}^{\mathrm{L}}\left|\mathrm{z}^{2}(\mathrm{x})\right| \mathrm{dx}} \quad \text { con } \mathrm{z}(\mathrm{x})=\mathrm{z}_{\mathrm{i}}-\overline{\mathrm{z}}
\end{aligned}
$$

siendo $\mathrm{L}$ la longitud de muestreo y $\mathrm{z}(\mathrm{x})$ la desviación en cada punto del perfil de rugosidad $\left(\mathrm{z}_{\mathrm{i}}\right)$ desde la línea media ( $\mathrm{z}$ ), calculada de manera que las áreas por encima y por debajo sean iguales. 


\section{RESULTADOS Y DISCUSIÓN}

\section{Comparación de chapas de desenrollo de distintas especies de madera por medida de ángulos de contacto}

En la tabla 2 se presentan los valores de los ángulos de velocidad de mojado constante (cwra) para las dos caras de chapas de madera obtenidas por desenrollo de las cinco especies estudiadas (ayous, chopo, okume, fromager y eucalipto) y con los cuatro líquidos de prueba empleados (agua, formamida, diyodometano y etilenglicol). En la figura 1 se muestra a modo de ejemplo la aplicación del método diferencial para el cálculo del cwra para la cara exterior de una chapa de madera de chopo en contacto con agua.

Para cada especie y líquido de prueba se han comparado los valores de cwra de las caras interna y externa de las chapas, siendo la cara interna la que entra en contacto con la cuchilla en el proceso de desenrollo. Para el chopo y el eucalipto el valor del cwra de la cara exterior es menor que el de la cara interior para todos los líquidos de prueba. En el caso del chopo la diferencia es significativa para todos los líquidos excepto para el etilenglicol, mientras que en el caso del eucalipto las diferencia sólo es significativa para la formamida. El comportamiento del okume es opuesto al del chopo y eucalipto con valores significativamente menores de cwra en la cara interna con todos los líquidos de prueba, a excepción de la formamida para la que no se han detectado diferencias. En el caso del ayous no se detectaron diferencias significativas entre el comportamiento de las dos caras para ninguno de los líquidos de prueba pero el menor valor de cwra se encontró en la cara interior o exterior dependiendo del líquido empleado. El fromager mostró un comportamiento análogo al ayous con menores valores de cwra en la cara exterior o interior dependiendo del líquido de prueba, siendo las diferencias significativas para agua y diyodometano. Resumiendo, para todos los líquidos de prueba empleados, las chapas de chopo y eucalipto presentan un mejor mojado por la cara exterior que por la interior, siendo el comportamiento a la inversa para las chapas de okume. En el caso de ayous y fromager el mojado depende del líquido empleado. Shupe et al. (1998) estudiando el efecto de la cara de la chapa en el mojado de chapas de pino también encontraron que su influencia dependía del líquido empleado siendo despreciable con una resina fenol-formaldehido pero crítico con agua para la que encontraron valores del ángulo de contacto significativamente más bajos por la cara interior que por la exterior.

Por otro lado, se observa que entre todas las especies de madera estudiadas, el okume es el que presenta una mayor capacidad de mojado con los menores valores de cwra para todos los líquidos de prueba, excepto el agua para la que el fromager presenta el menor valor en su cara exterior. Por el contrario, el fromager es la especie que muestra las peores propiedades de mojado al tener, salvo en el caso del agua, los mayores valores de cwra.

\section{Determinación de la energía libre superficial}

En la Figura 2 se presenta a modo de ejemplo la linearización de la ecuación 5 para la cara interior de una chapa de eucalipto. Los valores de la energía libre superficial $\left(\gamma_{\mathrm{SV}}\right)$ de todas las especies de madera estudiadas tanto por su cara exterior como interior, así como de sus componentes polar y dispersa, se muestran en la tabla 3 junto con los valores de los coeficientes de correlación $\mathrm{R}^{2}$ resultantes de la linearización de la ecuación 5.

La energía libre superficial $\left(\gamma_{\mathrm{sv}}\right)$ de las especies de madera estudiadas osciló entre 49,8-63,9 mJ/m², dentro del rango de valores encontrado para otras maderas duras 43,6-68,8 mJ/ $\mathrm{m}^{2}$ (Piao et al. 2010), y disminuyeron en el orden okume $>$ fromager $>$ chopo $>$ ayous $\sim$ eucalipto, lo que indica un descenso de la capacidad de mojado en ese mismo orden. El okume con el mayor valor de la energía libre superficial, como se explicó anteriormente, también presentó los menores valores del ángulo de contacto de equilibrio cwra, lo que la confirma como la especie de madera con las mejores propiedades de mojado entre las estudiadas. En todas las especies de madera, con la excepción del fromager, es mayor la componente dispersa que la componente polar de la energía libre superficial como también se ha encontrado para otras 
especies de maderas duras (Piao et al. 2010, de Meijer et al. 2000) y para la mayoría de los polímeros (Gindl y Tschegg 2002).

Tabla 2. Ángulos de velocidad de mojado constante (cwra) para las caras exterior y interior de chapas obtenidas por desenrollo de madera de ayous, chopo, okume, fromager y eucalipto en contacto con agua, formamida, etilenglicol y diyodometano.

\begin{tabular}{|c|c|c|c|c|c|}
\hline $\begin{array}{c}\text { Especie } \\
\text { madera }\end{array}$ & $\begin{array}{c}\text { Cara } \\
\text { chapa }\end{array}$ & $\begin{array}{c}\text { cwra } \mathbf{(}^{\mathbf{0}} \text { ) } \\
\text { Agua }\end{array}$ & $\begin{array}{c}\text { cwra } \mathbf{(}^{\mathbf{o}} \text { ) } \\
\text { Formamida }^{\mathrm{a}}\end{array}$ & $\begin{array}{c}\text { cwra } \mathbf{(}^{\mathbf{o}} \text { ) } \\
\text { Etilenglicol }\end{array}$ & $\begin{array}{c}\text { cwra } \mathbf{(}^{\mathbf{o}} \text { ) } \\
\text { Diyodometano }\end{array}$ \\
\hline Ayous & exterior & $50,98^{\mathrm{a}}(2,30)$ & $18,69^{\mathrm{a}}(3,13)$ & $27,80^{\mathrm{a}}(6,02)$ & $52,41^{\mathrm{a}}(3,09)$ \\
\hline Ayous & interior & $48,25^{\mathrm{a}}(0,70)$ & $18,42^{\mathrm{a}}(3,86)$ & $24,15^{\mathrm{a}}(2,14)$ & $54,73^{\mathrm{a}}(0,68)$ \\
\hline Chopo & exterior & $42,54^{\mathrm{b}}(7,21)$ & $27,56^{\mathrm{b}}(5,44)$ & $26,44^{\mathrm{b}}(3,63)$ & $47,92^{\mathrm{b}}(1,38)$ \\
\hline Chopo & interior & $51,24^{\mathrm{c}}(2,42)$ & $30,68^{\mathrm{c}}(1,94)$ & $29,07^{\mathrm{b}}(3,36)$ & $50,93^{\mathrm{c}}(0,89)$ \\
\hline Okume & exterior & $35,70^{\mathrm{d}}(2,16)$ & $10,21^{\mathrm{d}}(0,94)$ & $17,91^{\mathrm{c}}(3,69)$ & $33,72^{\mathrm{d}}(1,59)$ \\
\hline Okume & interior & $19,16^{\mathrm{e}}(1,89)$ & $10,43^{\mathrm{d}}(0,87)$ & $11,77^{\mathrm{d}}(1,35)$ & $25,85^{\mathrm{e}}(5,77)$ \\
\hline Fromager & exterior & $23,85^{\mathrm{f}}(0,44)$ & $32,56^{\mathrm{e}}(1,16)$ & $30,88^{\mathrm{e}}(6,26)$ & $64,91^{\mathrm{f}}(1,46)$ \\
\hline Fromager & interior & $29,14^{\mathrm{g}}(7,17)$ & $34,15^{\mathrm{e}}(3,78)$ & $27,57^{\mathrm{e}}(2,72)$ & $57,50^{\mathrm{g}}(6,88)$ \\
\hline Eucalipto & exterior & $47,34^{\mathrm{h}}(5,02)$ & $26,73^{\mathrm{f}}(2,17)$ & $23,98^{\mathrm{f}}(4,20)$ & $52,42^{\mathrm{h}}(6,61)$ \\
\hline
\end{tabular}

Media de 24 determinaciones ( Desviación standard)

apara cada especie y líquido de prueba, letras iguales indican que no hay diferencia significativa entre las medias de la cara exterior e interior para un nivel de confianza del $95 \%$.

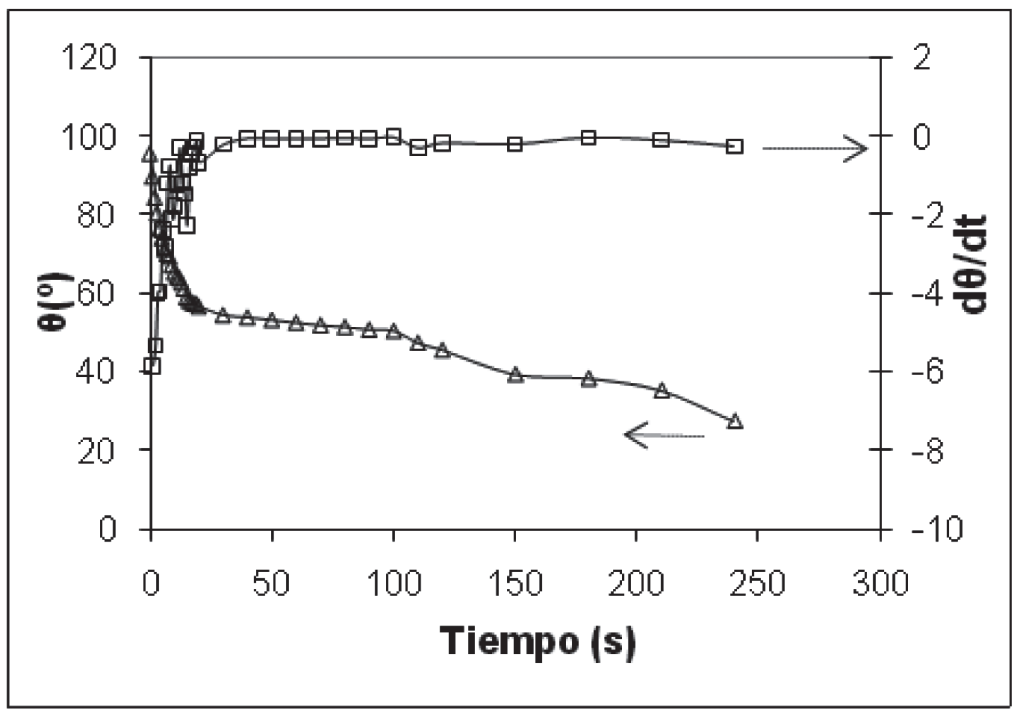

Figura 1. Evolución con el tiempo del ángulo de contacto y de su derivada, para la cara exterior de una chapa de madera de chopo en contacto con agua. 
Comparando los valores de la energía libre superficial de las dos caras de las chapas en general no se encontraron diferencias muy significativas, siendo el mayor cambio el del okume lo que concuerda con las mayores diferencias encontradas entre los ángulos de contacto de equilibrio de las caras interior y exterior para esta especie. Para chopo, eucalipto y fromager la energía libre superficial es mayor en la cara exterior que en la interior, lo que indica un mejor mojado en la primera y concuerda, en el caso del chopo y eucalipto, con los resultados obtenidos para cwra con menores valores para la cara exterior. Para ayous y okume, $\gamma_{\mathrm{SV}}$ es mayor en la cara interior lo que concuerda, en el caso del okume, con los valores obtenidos para cwra. Diversos factores tales como diferencias en la porosidad de la madera, rugosidad superficial o la heterogeneidad química de la superficie, en particular la presencia de extractos pueden ser los responsables de las diferencias observadas (de Meijer et al. 2000).

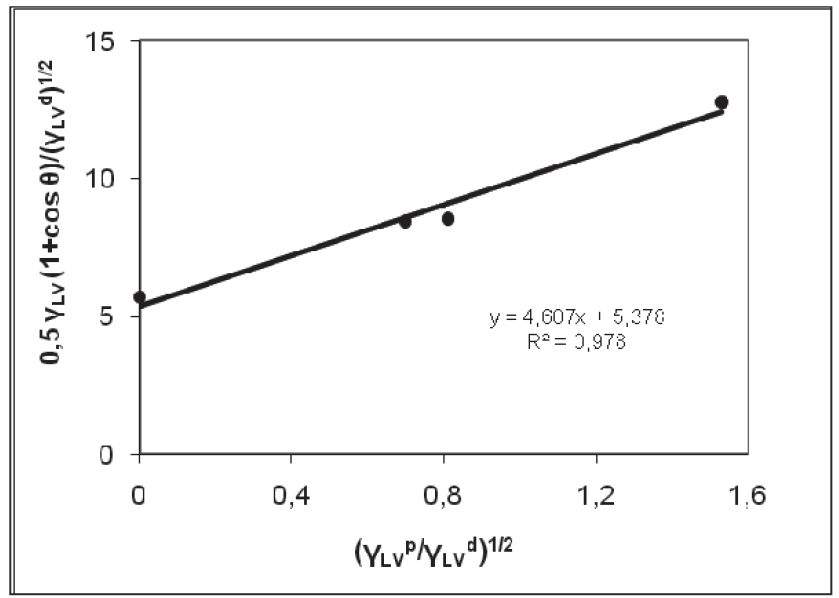

Figura 2. Aplicación del método de Owens-Wendt-Rabel-Kaelble para la cara interior de una chapa de eucalipto.

Tabla 3. Valores de la energía libre superficial $\left(\gamma_{\mathrm{SV}}\right)$ y de sus componentes polar $\left(\gamma_{\mathrm{SV}}{ }^{\mathrm{p}}\right)$ y dispersa $\left(\gamma_{\mathrm{SV}}{ }^{\mathrm{d}}\right)$ (ecuación 6) para las cara exterior y interior de chapas de ayous, chopo, okume, fromager y eucalipto.

\begin{tabular}{|l|c|c|c|c|c|}
\hline $\begin{array}{c}\text { Especie } \\
\text { madera }\end{array}$ & $\begin{array}{c}\text { Cara } \\
\text { Chapa }\end{array}$ & $\begin{array}{c}\gamma_{\mathbf{S V}} \\
\left(\mathbf{m J} / \mathbf{m}^{2}\right)\end{array}$ & $\begin{array}{c}\gamma_{\mathbf{S v}}{ }^{\mathbf{d}} \\
\left(\mathbf{m J} / \mathbf{m}^{2}\right)\end{array}$ & $\begin{array}{c}\gamma_{\mathbf{S v}}{ }^{\mathbf{p}} \\
\left(\mathbf{m J J} / \mathbf{m}^{2}\right)\end{array}$ & $\mathbf{R}^{\mathbf{2}}$ \\
\hline Ayous & Exterior & 51,0 & 30,7 & 20,3 & 0,97 \\
\hline Ayous & Interior & 52,1 & 29,3 & 22,8 & 0,98 \\
\hline Chopo & Exterior & 53,7 & 29,3 & 24,4 & 0,95 \\
\hline Chopo & Interior & 49,8 & 29,9 & 19,9 & 0,97 \\
\hline Okume & Exterior & 59,0 & 34,6 & 24,4 & 0,93 \\
\hline Okume & Interior & 63,8 & 34,0 & 29,9 & 0,90 \\
\hline Fromager & Exterior & 59,9 & 18,8 & 41,2 & 0,95 \\
\hline Fromager & Interior & 57,7 & 22,2 & 35,4 & 0,94 \\
\hline Eucalipto & Exterior & 52,0 & 29,2 & 22,8 & 0,98 \\
\hline Eucalipto & Interior & 50,2 & 28,9 & 21,2 & 0,98 \\
\hline
\end{tabular}




\section{Caracterización superficial de las chapas por microscopía de barrido láser confocal (CLSM)}

Mediante CLSM se han analizado las caras exterior y interior de chapas de madera de ayous, chopo, okume, fromager y eucalipto. En las figuras 3 y 4 se presentan, a modo de ejemplo, para uno de los campos analizados de una cara exterior de una chapa de ayous, la reconstrucción topográfica 3D y la imagen topográfica de la superficie. Los parámetros de rugosidad Ra y RMS calculados según la norma DIN EN ISO 4287, empleando las ecuaciones 7 y 8 , se muestran en la tabla 4 para las caras exterior y interior de las chapas de todas las especies de madera estudiadas.

Tabla 4. Parámetros de rugosidad Ra y RMS calculados según la norma DIN EN ISO 4287 para las caras exterior y interior de chapas de ayous, chopo, okume, fromager y eucalipto.

\begin{tabular}{|l|c|c|c|}
\hline $\begin{array}{c}\text { Especie } \\
\text { madera }\end{array}$ & $\begin{array}{c}\text { Cara } \\
\text { chapa }\end{array}$ & $\begin{array}{c}\text { Ra } \\
(\boldsymbol{\mu m})\end{array}$ & $\begin{array}{c}\text { RMS } \\
(\boldsymbol{\mu m})\end{array}$ \\
\hline Ayous & exterior & $27,38(8,44)$ & $37,37(13,89)$ \\
\hline Ayous & interior & $21,96(6,22)$ & $30,79(8,51)$ \\
\hline Chopo & exterior & $33,31(8,71)$ & $43,54(11,07)$ \\
\hline Chopo & interior & $21,22(2,71)$ & $29,41(2,70)$ \\
\hline Okume & exterior & $21,99(4,68)$ & $31,93(5,33)$ \\
\hline Okume & interior & $34,34(8,97)$ & $47,49(11,05)$ \\
\hline Fromager & exterior & $33,66(14,31)$ & $46,35(21,53)$ \\
\hline Fromager & interior & $32,39(15,42)$ & $41,68(18,01)$ \\
\hline Eucalipto & exterior & $15,75(4,66)$ & $24,62(5,07)$ \\
\hline Eucalipto & interior & $20,62(8,70)$ & $29,31(9,29)$ \\
\hline
\end{tabular}

Media de 5 determinaciones (desviación estándar)

Los menores valores de Ra y RMS se han obtenido para la cara exterior del eucalipto y los mayores para la cara interior del okume, que es precisamente la que presentó el mayor valor de la energía libre superficial y los menores ángulos de contacto de equilibrio de entre todas las analizadas. Este resultado concuerda con los obtenidos por otros autores que relacionan una mayor rugosidad superficial de la madera con menores ángulos de contacto y mejores propiedades de mojado (Piao et al. 2010).

Comparando los parámetros de rugosidad para las dos caras de una chapa se encontró que los de la cara exterior son mayores que los de la interior para las especies ayous, chopo y fromager ocurriendo lo contrario para okume y eucalipto. En el caso del okume y el chopo la cara que presenta los mayores valores de Ra y RMS, la cara interior o exterior, respectivamente, es la que presenta el mayor valor de la energía libre superficial y los menores valores del ángulo de contacto de equilibrio cwra o lo que es lo mismo la mayor capacidad de mojado, no existiendo dicha relación para el resto de las especies. 


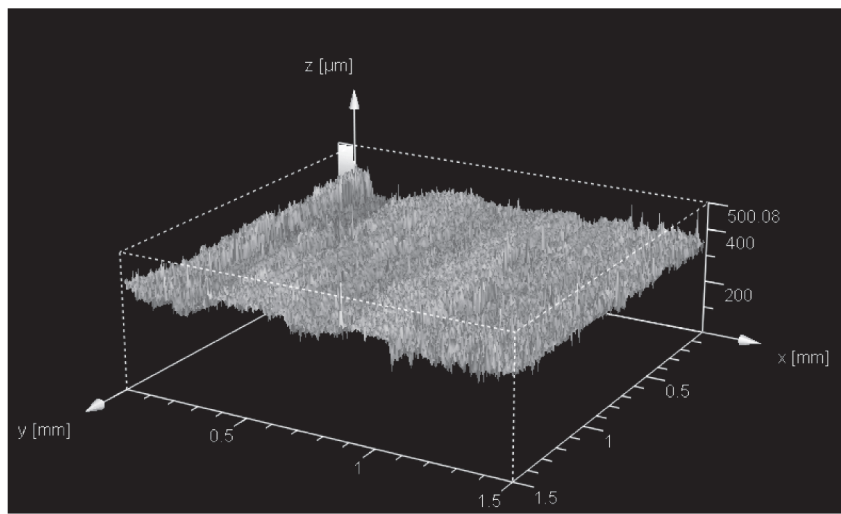

Figura 3. Reconstrucción topográfica 3D de uno de los campos analizados por CLSM de la cara exterior de una chapa de ayous.

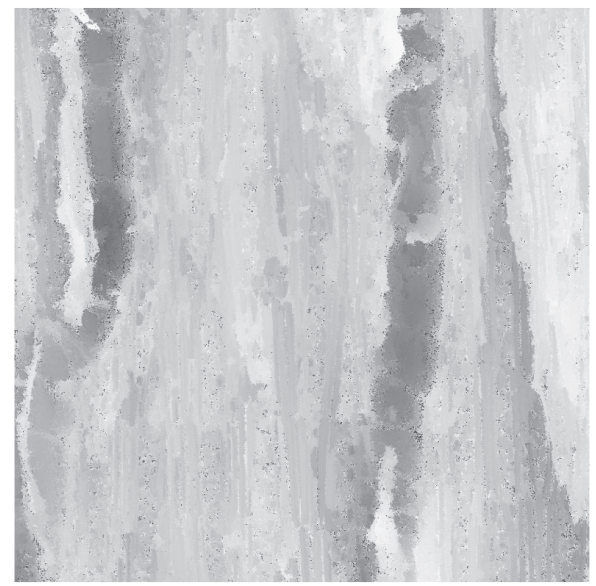

Figura 4. Imagen topogrática de la superticie de uno de los campos analizados por CLSM de la cara exterior de una chapa de ayous.

\section{CONCLUSIONES}

De todas las especies de madera estudiadas: ayous, chopo, okume, fromager y eucalipto, el okume presentó los menores valores de los ángulos de contacto de equilibrio con los diferentes líquidos de prueba utilizados y el fromager los mayores.

La energía libre superficial $\left(\gamma_{\mathrm{SV}}\right)$ de las especies de madera estudiadas osciló entre $49-64 \mathrm{~mJ} / \mathrm{m}^{2}$ y disminuyó en el orden okume $>$ fromager $>$ chopo $>$ ayous $\sim$ eucalipto, lo que indica un descenso de la capacidad de mojado en ese mismo orden. En todas las especies de madera, con la excepción del fromager, es mayor la componente dispersa que la componente polar de la energía libre superficial.

No se encontraron diferencias muy significativas entre los valores de la energía libre superficial de las dos caras de las chapas, siendo el mayor cambio el del okume que también mostró, en general, las mayores diferencias entre los ángulos de contacto de equilibrio de ambas caras.

El okume presentó el mayor valor de la energía libre superficial, los menores ángulos de contacto de equilibrio y los mayores valores de los parámetros de rugosidad superficial, lo que la convierten en la especie con las mejores propiedades de mojado entre todas las analizadas. 


\section{BIBLIOGRAFÍA}

DIN EN ISO 4287. 1997. Especificación geométrica de productos (GPS) - Calidad superficial: Método del perfil. Términos, definiciones y parámetros del estado superficial.

de Meijer, M.; Haemers, S.; Cobben, W.; Militz, H. 2000. Surface energy determinations of wood: comparison of methods and wood species. Langmuir 16: 9352-9359.

Gindl, M ; Sinn, G. ; Reiterer, A. ; Tschegg, S. 2001. Wood surface energy and time dependence of wettability: A comparison of different wood surfaces using and acid-base approach. Holzforschung 55: 433-440.

Gindl, M ; Tschegg, S. 2002. Significance of the acidity of wood to the surface free energy components of different wood species. Langmuir 18: 3209-3212.

Kaelble, D.H. 1970. Dispersion-Polar Surface Tension Properties of Organic Solids. Journal of Adhesion 2 (2): 66-81.

Liptákova, E.; Kúdela, J.; Bastl, Z.; Spirovová, I. 1995. Influence of mechanical surface treatment of wood on the wetting process. Holzforschung 49: 369-375.

Nussbaum, R.M. 1999. Natural surface inactivation of scots pine and Norway spruce evaluated by contact angle measurements. Holz Roh Werkstoff 57 (6): 419-424.

Owens D.K.; Wendt, R.C. 1969. Estimation of the surface free energy of polymers. Journal of Applied Polymer Science 13 (8): 1741-1747.

Piao, CH.; Winandy, J.E.; Shupe, T.F. 2010. From Hydrophilicity to Hydrophobicity: A Critical Review: Part I. Wettability and Surface Behavior. Wood and Fiber Science 42(4): 490-510.

Scheikl, M. ; Dunky, M. 1998. Measurement of dynamic and static contact angles on wood for the determination of its surface tension and the penetration of liquids into the wood surface. Holzforschung $52: 89-94$

Shupe, T. E.; Hse, CH.Y.; Choong, E.T.; Groom, L.H. 1998. Effect of wood grain and veneer side on loblolly pine veneer wettability. Forest Product J. 46(6): 95-97.

Tshabalala M.A. 2005. Surface Characterization. En: Handbook of Wood chemistry and Wood composites. Rowell R.M. Ed. CRC Press, Boca Ratón, 187-211. 